\title{
Proteomic analyses using an accurate mass and time tag strategy
}

\author{
Ljiljana Paša-Tolić, Christophe Masselon, Richard C. Barry, Yufeng Shen, and Richard D. Smith
}

BioTechniques 37:621-639(October 2004)

\begin{abstract}
An accurate mass and time (AMT) tag approach for proteomic analyses has been developed over the past several years to facilitate comprehensive high-throughput proteomic measurements. An AMT tag database for an organism, tissue, or cell line is established by initially performing standard shotgun proteomic analysis and, most importantly, by validating peptide identifications using the mass measurement accuracy of Fourier transform ion cyclotron resonance (FTICR) mass spectrometry (MS) and liquid chromatography $(L C)$ elution time constraint. Creation of an AMT tag database largely obviates the need for subsequent MS/MS analyses, and thus facilitates high-throughput analyses. The strength of this technology resides in the ability to achieve highly efficient and reproducible one-dimensional reversed-phased LC separations in conjunction with highly accurate mass measurements using FTICR $M S$. Recent improvements allow for the analysis of as little as picrogram amounts of proteome samples by minimizing sample handling and maximizing peptide recovery. The nanoproteomics platform has also demonstrated the ability to detect $>10^{6}$ differences in protein abundances and identify more abundant proteins from subpicogram amounts of samples. The AMT tag approach is poised to become a new standard technique for the in-depth and high-throughput analysis of complex organisms and clinical samples, with the potential to extend the analysis to a single mammalian cell.
\end{abstract}

\section{INTRODUCTION}

Recent technological advances have allowed complex biological systems to be studied from a global perspective, whereby a great many components are monitored in parallel. For example, one can simultaneously study the interplay of large numbers of biomolecules under the influence of a particular stimulus in order to decipher the critical entities regulated in response to that stimulus. These advances hold promise for revealing disease mechanisms and identifying biomarkers to facilitate the development of drug therapeutics (1).

Genomics and proteomics are complementary global approaches used to understand, interpret, and identify the molecular processes that influence biological systems. While the genome of an organism may be considered static, gene expression is a dynamic event that is constantly influenced by environmental and physiological conditions. Thus, for complex biological systems, a wealth of information can be gained by monitoring the actual gene products (i.e., mRNA and proteins) being expressed to maintain cellular function. Tracking transcript levels exclusively, although currently easier, has limitations for understanding the mechanisms of biological processes. Recent detailed studies have shown that mRNA levels do not necessarily correlate well with protein abundance, presumably because different mRNAs exhibit different rates of translation and turnover (2-5). Furthermore, the amount of functional protein (as translated by the mRNA) may be essentially independent of mRNA levels altogether as a result of posttranslational modifications (PTMs), alternative splicing, or differential rates of protein turnover. Thus, measuring both mRNA and protein abundances provides more comprehensive information about the macromolecules most intimately involved in cellular regulation and operation.

An essential component for establishing a comprehensive model of any biological system is the accurate iden- tification and quantification of protein abundances at any given time. The challenges for proteomics include identifying all proteins expressed under different conditions (e.g., in health and disease) and characterizing their modifications, interactions, and structure. Such information can provide a basis for understanding genetic variants, gene functions, and the mechanisms of action needed to develop the means to diagnose, treat, and protect against disease.

Proteomic measurement techniques can generally be divided into predominantly gel-based and solution-based (i.e., gel-free) approaches. Gel-based approaches, such as two-dimensional gel electrophoresis (2-DE), typically employ matrix-assisted laser desorption/ionization (MALDI) mass spectrometry (MS) (6) and peptide mass fingerprinting (7) for the identification of gel-separated proteins. Solutionbased approaches generally utilize liquid chromatography (LC) separations with electrospray ionization (ESI) tandem MS (MS/MS) (8) for the identifi- 
cation of peptides obtained by tryptic digestion of complex protein mixtures such as whole cell lysates $(9,10)$. While both of these approaches are invaluable for identifying proteins, they still suffer from significant limitations, a key element being the lack of throughput necessary to address the numerous samples required to study the ever-changing proteome of an organism in response to a variety of stimuli.

Over the past several years, we have developed a new approach for proteomics, which combines the high resolution and mass measurement accuracy (MMA) of Fourier transform ion cyclotron resonance (FTICR), high-efficiency capillary LC separations, and putative amino acid sequence information obtained by MS/MS to enable comprehensive high-throughput measurements (11-13). This so-called accurate mass and time (AMT) tag approach provides high-quality quantitative data on protein identity and abundance at higher sensitivity and over a greater range of cellular abundances, without the need to perform repetitive MS/MS analyses, thereby dramatically improving the throughput of proteomic measurements. Building upon initial success, we have further extended the AMT tag approach for nanoscale sample processing and analysis, which we refer to as "nanoproteomics." Presently, this nanoproteomics platform can be used for comprehensive proteome analyses of nanogram quantities of material and allows for the characterization of highly abundant proteins in picogram size samples, indicating the potential to characterize the proteins present in a single mammalian cell.

\section{RATIONALE FOR A NEW PROTEOMICS TECHNOLOGY}

The ideal proteomic method would detect all proteins (including PTMs) that are present at a few copies per cell to millions of copies per cell, allow identifications to be made from complex mixtures with statistical confidence by incorporating multiple metrics or redundancy in sequence coverage, and be amenable to quantitative methods, high-throughput analyses, and automation.

\section{Limited Throughput and Coverage of Existing Technologies}

Although a great number of proteomic studies still rely on 2-DE, alternative technologies based on liquid phase separations of proteolytically digested peptides and tandem MS (also known as shotgun proteomics) have recently gained significant momentum $(14,15)$. Conventionally, 2-DE methodologies involve separate extraction, digestion, and identification of each protein (spot) using mass spectrometric methods $(16,17)$. Advantages of these approaches that provide "global" snapshots of a proteome include the capability to resolve protein isoforms and PTMs. However, these methodologies suffer from significant drawbacks associated with the separation method and staining procedures, such as the inability to analyze proteins with extreme isoelectric points (pIs) or molecular masses $\left(M_{\mathrm{r}} \mathrm{s}\right)$, limited throughput despite significant automation efforts, relatively large sample requirements (50-200 $\mu \mathrm{g}$ total protein per analysis), inadequate detection limits (0.1-10 ng protein/spot), and narrow dynamic range $\left(<10^{3}\right)(18)$. Thus, 2-DE is biased toward the more abundant proteins being expressed that have compatible pI (3-10) and $M_{\mathrm{r}}(15-150 \mathrm{kDa})$ (19). Furthermore, solubility issues limit the ability to characterize many membrane-associated proteins at the intact protein level.

Present shotgun proteomic strategies are based on the analysis of complex peptide mixtures, using chromatographic methods to separate the sample components (thereby reducing sample complexity) (20) and ESI-MS/MS to obtain amino acid sequence information for identifying proteins (21). These approaches circumvent many of the problems associated with 2-DE $(9,22-$ 24) but still fall short of realizing the high-throughput necessary for many applications. One of the weaknesses of using a shotgun proteomics strategy is the need to re-identify peptides by MS/ MS in each analysis. In addition, the MS/MS approach is effectively blind to peptides that do not get selected for dissociation, which is a significant fraction of the total peptides in a complex proteomic mixture, despite pre- fractionation (25). In an effort to speed analyses and expand the coverage of proteomic experiments, we have developed an MS-based method that exploits the high mass accuracy, sensitivity, and resolution of FTICR to identify peptide markers that can then be used to reconfirm peptides in subsequent experiments, without the need for repetitive MS/MS measurements. While LC-MS/MS proteome analyses are still employed for initial peptide identification, such extensive analyses need only be performed once for a particular biological system and serve to create an effective "look-up table" of markers for identifying peptides using their characteristic accurate masses and LC elution times. These peptide markers or AMT tags can then be used with subsequent high accuracy mass and normalized LC elution time measurements to identify many peptides in each spectrum without MS/MS, providing both greater sensitivity and increased throughput.

A key attraction of this approach is a significantly increased throughput for subsequent quantitative measurements of changes in protein abundances, based upon predetermined AMT tags and the use of stable-isotope labeling or other approaches for comparative analyses. For example, conventional ion trap MS instrumentation generates on the order of $4000 \mathrm{MS} / \mathrm{MS}$ spectra in a single LC analysis, with typically less than $10 \%$ of acquired MS/MS spectra resulting in confident peptide identifications. On average, we were able to identify approximately 250 peptides [corresponding to $4 \%$ coverage of the microbial (e.g., Deinoccocus radiodurans) proteome] in a single 2.5-hours-long LC-MS/MS analysis using an ion trap. However, by using the high efficiency of reversedphase LC coupled with FTICR, with a combined peak capacity of $>10^{7}$, we detected, on average, 25,000 to 50,000 unique isotopic distributions, representing peptides that are possibly identifiable as AMT tags in an LC-FTICR analysis of the same (e.g., D. radiodurans) whole cell lysate. When coupled with stable-isotope labeling, this setup gave approximately $20 \%$ proteome coverage for a single approximately 2.5-hourslong LC-FTICR analysis. In addition, recent advances in FTICR instrumentation allowed approximately $40 \%$ of the 
D. radiodurans microbial proteome to be detected and quantified in a single LC-FTICR analysis (26).

\section{AMT Tags for Peptide Identifications}

The power of MS for protein identification derives from the specificity of mass measurements for either the intact peptides (MS) or their fragments (after dissociation in MS/MS) and is implicitly based upon the relatively limited number of peptide sequences associated with an organism in comparison with the total number of pos- sible amino acid combinations. For example, the number of potentially mass distinguishable 30-mer tryptic peptides, estimated from the number of possible combinations, is $>10^{13}$, compared to the much smaller number of 30-mer tryptic peptides from a specific organism (e.g., 3602 for Caenorhabditis elegans). Thus, given sufficient MMA, a peptide mass measurement can often be confidently attributed to a single protein within the constraints provided by a single genome sequence and its predicted proteome. Armed with this notion, we can evaluate the level of MMA that would be sufficient to

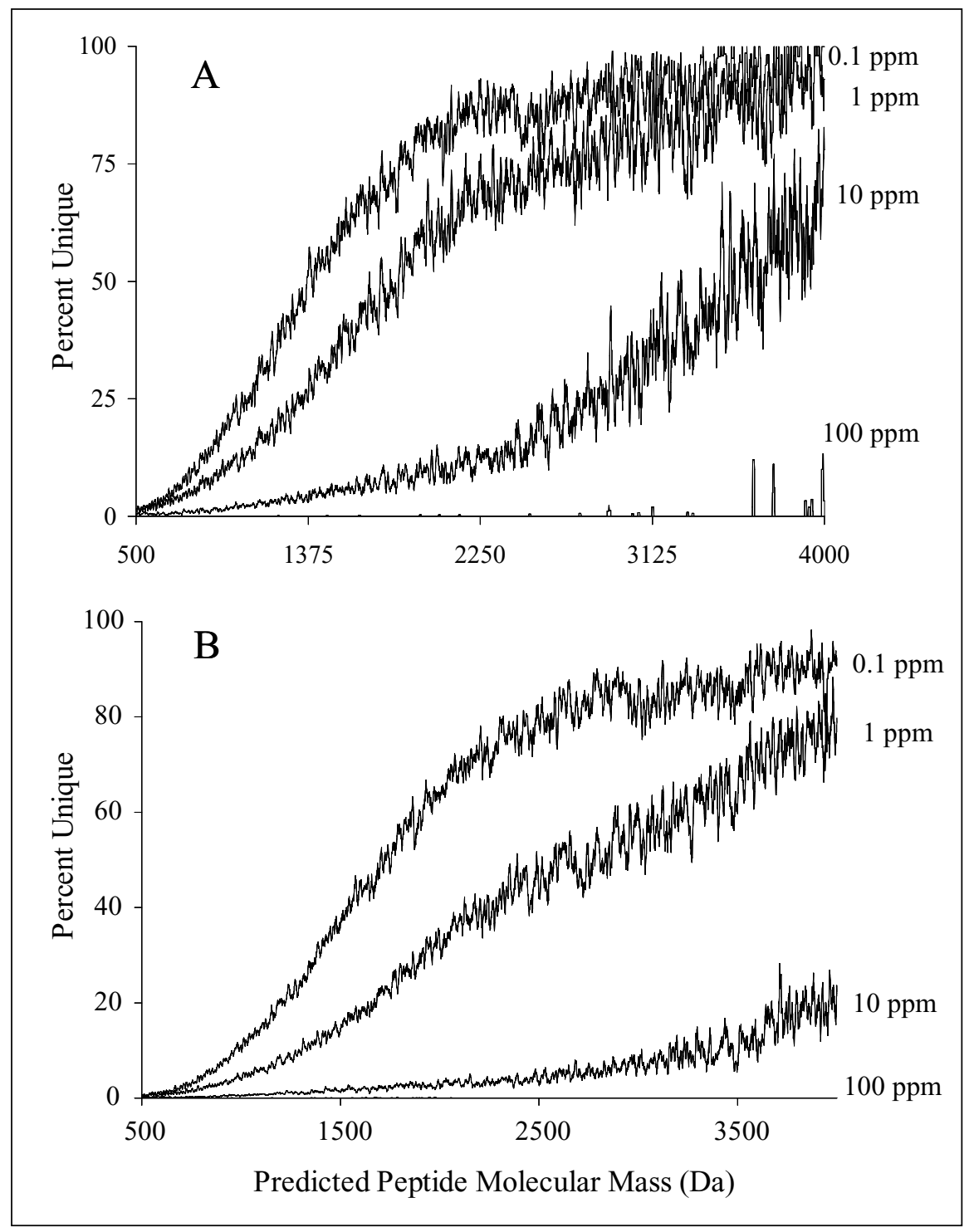

Figure 1. Calculated percent unique (distinctive) tryptic fragments as a function of tryptic fragment mass at four different levels of mass measurement accuracy for the predicted proteins of (A) yeast and (B) Caenorhabditis elegans. Reproduced with permission from Reference 27. 
identify a peptide (and its corresponding protein) in the context of a given organism. Simulations using the Saccharomyces cerevisiae and C. elegans full genome databases indicate that at MMA $<10 \mathrm{ppm}$, a sizable fraction of the tryptic peptides predicted for these organisms can act as confident peptide markers for their corresponding protein (Figure 1). At $1 \mathrm{ppm}$, this fraction becomes approximately $50 \%$ for yeast and approximately $40 \%$ for C. elegans, covering $98 \%$ and $96.6 \%$ of the predicted proteome of these organisms, respectively (27). In general, the greater the MMA, the greater the specificity of the mass and the more confident the identification.

While MS provides the mass of the observed species, uncertainties with respect to their identity can arise due to noise, contaminants, or species not accounted for by the predicted proteome, such as PTMs. Therefore, additional information is desirable in order to make as confident an identification as possible. Additional confidence is gained when the observed species have already been identified at least once by MS/MS. Moreover, the use of high-efficiency capillary LC brings about another characteristic of the peptides under study - their retention time-that can be used in combination with an accurate mass as an additional metric to make more reliable identifications.

We therefore conclude that a considerable number of peptides identified by MS/MS can act as protein markers based on the uniqueness of a peptide's mass and retention time in the context of a given proteome. Once such a set of AMT tags has been identified for a given organism, there is no need for repetitive MS/MS identification, and all previously identified species can potentially be revealed in a single LC-FTICR acquisition. This single-acquisition ability is the key to increasing the throughput, sensitivity, and dynamic range of proteomic measurements.

\section{Potential for Quantitative Studies of Global Proteomes}

Several recent reviews discuss current approaches to quantitative analysis using shotgun proteomics (28-30). Figure 2 summarizes the various quantitative strategies for LC-based proteomic methods. Most approaches incorporate the use of stable-isotope labeling to obtain relative quantitation of expressed species. These methods involve mixing (at either the whole cell, protein, or peptide level) and simultaneous analysis of two samples distinctively labeled by stable isotopes that can be sufficiently resolved in the mass spectrum. Peptide and protein abundance ratios are typically determined by calculating the ratio of mass spectral peak intensities over the elution peak areas for the two versions of a peptide. Examples of methodologies include metabolic labeling using ${ }^{14} \mathrm{~N} /{ }^{15} \mathrm{~N}$ (Figure 2A) or other stable-isotope enriched or depleted media, tagging of cysteine residues us-

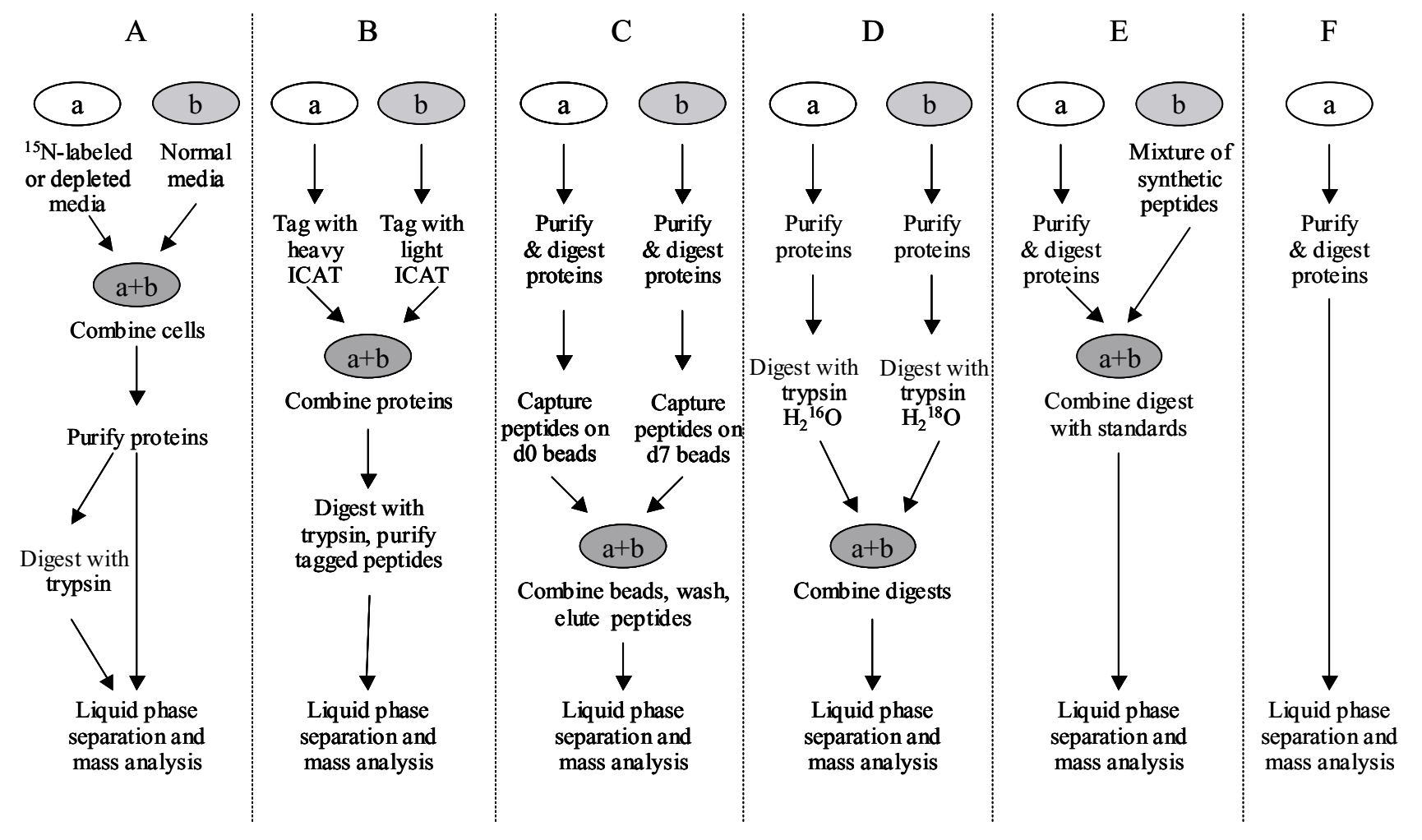

Figure 2. Contemporary gel-free approaches for quantitative proteomics. These include strategies for relative quantitation, such as (A) metabolic labeling, (B) isotope-coded affinity tags (ICATs), (C) solid-phase ICAT, (D) differential ${ }^{16} \mathrm{O} /{ }^{18} \mathrm{O}$ labeling during tryptic digestion, and methods for absolute quantitation such as using $(\mathrm{E})$ the addition of standards or $(\mathrm{F})$ direct use of peptides abundances to derive proteins concentrations. 
ing isotope-coded affinity tags (ICATs; Figure 2B), extraction, and simultaneous tagging of cysteine-containing peptides by solid-phase ICAT (Figure 2C), and ${ }^{16} \mathrm{O} /{ }^{18} \mathrm{O}$ labeling during proteolytic digestion (Figure 2D) to obtain precise measures of the relative abundances of proteins (22,28-38). Methods for determining the absolute abundances of proteins involve either spiking with known amounts of internal standards (Figure 2E) [e.g., isotopically labeled synthetic peptides (39)] or directly evaluating the protein concentrations based on the (corrected) abundance of their detected peptides (Figure 2F). Although these methods offer great promise, both are quite involved and are not currently widely applied.

The crucial advantage of combining the LC-FTICR-based AMT tag approach with various stable isotopic labeling strategies is that it provides the resolving power and increased throughput necessary to obtain quantitative measurements of protein abundances. In this regard, the high-throughput of the AMT tag method is superior to other quantitative approaches. For very complex samples, such as the mixture of peptides from a whole cell lysate, a single LC-FTICR analysis can yield more information than a conventional LC-MS/MS experiment because a large fraction of the previously characterized peptides are amenable to AMT tagbased identification and quantitation in a single acquisition.

\section{REALIZATION OF THE AMT TAG APPROACH}

\section{Initial Implementation}

In the initial implementation of the AMT tag methodology, a newly designed very high-pressure LC system (12) was coupled to a conventional ion trap mass spectrometer (Finnigan LCQ $^{\mathrm{TM}}$; Thermo Finnegan, San Jose, CA, USA) for peptide identification by MS/MS using a Sequest $\mathrm{t}^{\mathrm{TM}}$-based search against genome sequence-derived databases (40-42). Extensive sample fractionation and a multi-run mass-to-charge ratio $(\mathrm{m} / \mathrm{z})$-segmented MS/MS strategy (43) were used to increase the number of peptide identifications. Subsequent experiments were carried out using an identical nano-capillary LC system coupled to an FTICR operated in MS-only mode to obtain the highest achievable sensitivity, mass accuracy, and dynamic range. A key attraction of this approach is its potential to significantly increase throughput for subsequent quantitative measurements of changes in protein abundances (following perturbation), based upon predetermined AMT tags and the use of stable-isotope labeling (i.e., metabolically, as genes are being expressed, or after biosynthesis). Figure 3 illustrates the two-stage strategy for exploiting high-resolution capillary LC separations integrated with the capabilities offered by FTICR to validate peptide AMT tags using accurate mass measurements combined with MS/MS identifications (stage 1). The AMT tags provide the basis for second-stage highthroughput studies without the need for repetitive MS/MS measurements (Figure 3 , stage 2 ).
The overall LC-MS effective resolution $(R)$ or peptide-distinguishing capability of the two-dimensional analysis can be expressed as:

$$
R=\frac{1}{M M A \times T M A}
$$

where the MMA and LC time measurement accuracy (TMA) provide essentially equivalent contributions for distinguishing peptides. The maximum $R$ that is presently achievable using onedimensional (1-D) LC separation is approximately $10^{8}$ (based upon $1 \mathrm{ppm}$ MMA and 0.01 TMA). Thus, the overall resolution statistically yields a distinguishing power equivalent to that approximately achievable with $0.01 \mathrm{ppm}$ MMA using only MS, but with different selectivity as the MMA $\times$ TMA product reflects both peptide chemical composition and its physicochemical properties. For microbial proteomics, the overall $0.5 \mathrm{ppm} 2$-D specificity $(10$ ppm MMA and 0.05 TMA) was proven

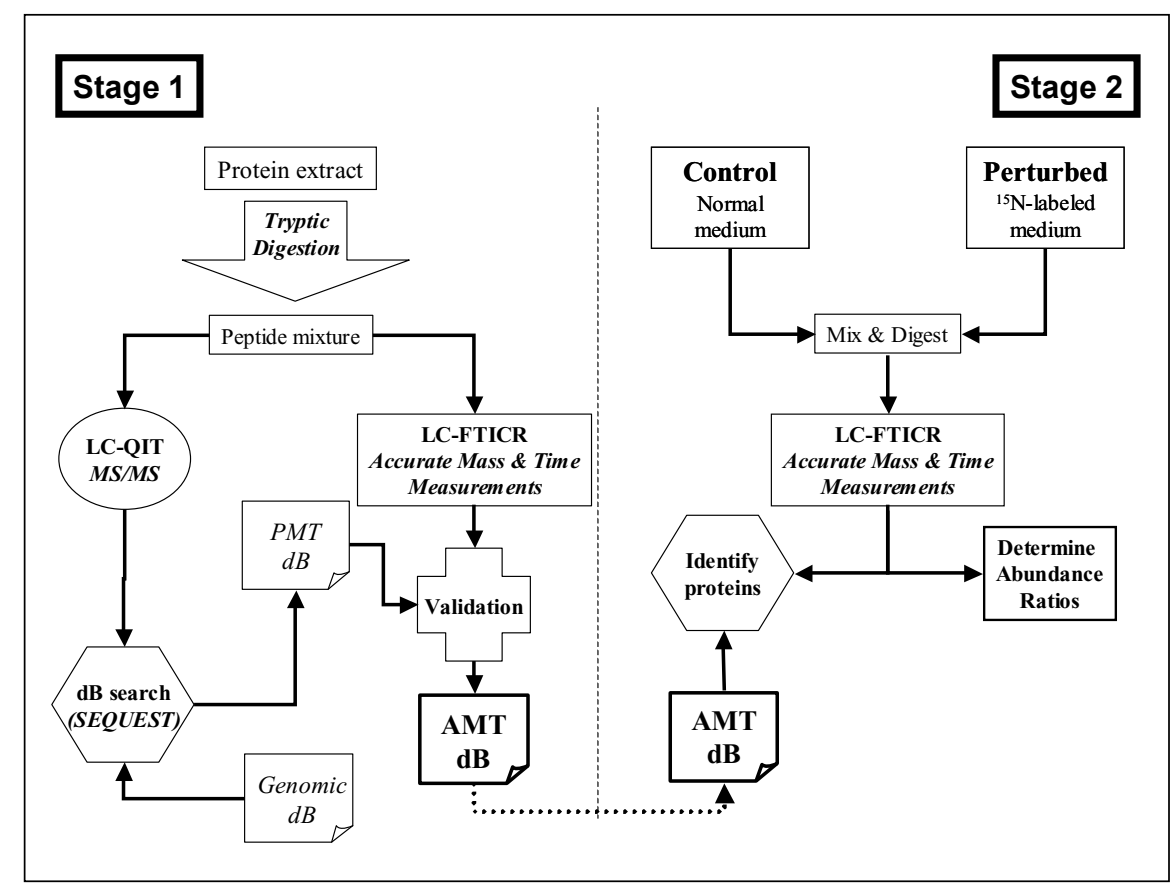

Figure 3. Experimental steps involved in accurate mass and time (AMT) tag-based proteomics. Stage 1: a proteome sample is analyzed by liquid chromatography tandem mass spectrometry (LC-MS/ MS) (e.g., using a conventional ion trap mass spectrometer), and peptides are identified by the resulting peptide fragmentation pattern as potential mass and time (PMT) tags. A similar proteome sample is analyzed under the same LC conditions using a Fourier transform ion cyclotron resonance (FTICR) MS-only analysis. An AMT tag is established when a peptide eluting at the same time and corresponding to the calculated mass (e.g., within $1 \mathrm{ppm}$ ) of the PMT tag initially identified is observed. By analyzing a plethora of samples, an AMT tag database is constructed. Stage 2: AMT tag peptides then function as markers to identify their corresponding proteins in all subsequent experiments analyzing a proteome sample from a specific organism, such as the protein level gene expression profiling illustrated here (adapted from Reference 73). dB, database; LC-QIT, liquid chromatography-quadrupole ion trap. 
sufficient to distinguish most peptides in even the most congested mass $\times$ time region and was typically used for peptide identification.

To achieve the desired TMA and account for the variability associated with high-pressure capillary LC separations using syringe pumps, packed columns, and very low flow rates, we sought an "intelligent" algorithm that could be used to compensate for run-to-run LC differences. Our initial analyses showed that the corrected LC elution time of a complex mixture of peptides could be as precise as approximately $1 \%$ after using retention time calibrants. Thus, we developed an approach for normalizing the retention times of all peptides to within the range $[0,1]$ by using an artificial neural network (ANN)-based algorithm. An initial evaluation of the linear algorithm used to standardize retention times showed that the standard deviation was below $\pm 2 \%$ (44). ANN technology aids in collating data from multiple LC runs and greatly enhances the AMT tag assignments established for peptides from LC-MS data.

The AMT tag approach was initially demonstrated in a global analysis of the prokaryote $D$. radiodurans $\mathrm{R} 1$ cultured under a number of different growth conditions. Over $60 \%$ of the proteome for the microbe was initially characterized (45), and a recent update of our $D$. radiodurans AMT tag database using refined data processing and additional experimental data increased the proteome coverage to over $80 \%$ of the total possible proteins. This level of comprehensive proteome coverage exceeded that achieved for any other organism to date and allowed $15 \%$ to $25 \%$ of the $D$. radiodurans proteome to be identified from AMT tags in a single LC-FTICR analysis compared to approximately $4 \%$ coverage typically feasible in a single LC-MS/MS analysis using an ion trap (45).

The key for the successful appli- cation of the AMT tag approach lies in the combined MMA and TMA, and it is likely that automation of the LC-FTICR experiment will allow more robust operations at higher throughput. Indeed, we have recently automated an LC system coupled to a commercial Apex III instrument (Bruker Daltonics, Billerica, MA, USA) and realized expected gains in throughput, robustness, and reliability (46).

\section{Extension of the AMT Tag Approach to More Conventional Technologies}

Central to the AMT tag approach are the MMA and TMA achieved. Recent advances in hybrid quadrupole time-offlight (qTOF) and TOF/TOF instrumentation suggest that similar strategies are feasible using TOF-MS technology (although adapted for the presently lower performances) (47). We envision extension of the AMT tag approach to additional instruments with high MMA and 
the incorporation of software to normalize peptide-LC retention time for more accurate TMA values (currently under development in our laboratory). Although modern TOF-MS has demonstrated MMA on the level of $5 \mathrm{ppm}$ (using internal calibration and given a signal sufficiently large to provide reasonable peak shape), this MS technology is still constrained by the attainable dynamic range. Commercially available FTICR instrumentation, such as the Finnigan LTQ FT ${ }^{\mathrm{TM}}$ (Thermo Finnegan) and Apex III instrument, is becoming increasingly more accessible to the proteomics community, providing the desired combination of increased sensitivity, high MMA, and extended dynamic range.

\section{Toward AMT Tag-Based Nano- proteomics for Clinical Studies}

To fulfill its promise for clinical applications, proteomics needs to address the often extremely small amounts of material available for analysis (e.g., microdissected tissues and biopsies), while simultaneously providing as much comprehensiveness, high-throughput, wide dynamic range coverage, and precise quantitation as possible. The AMT tag approach using small-diameter capillary LC-FTICR significantly decreases the amount of sample needed for analysis and simultaneously provides increased throughput. However, there is still a pressing need to develop robust, ultrasensitive techniques for routine labeling and characterization of samples of sizes below those of existing sample handling capabilities. Addressing this challenge has led to the development of new nanoscale sample processing techniques and LC-MS methods. Nanoproteomics measurements using picogram to nanogram sized total protein samples are qualitatively distinguishable from traditional proteomic studies (that use 10 $\mu \mathrm{g}$ to $1 \mathrm{mg}$ sample sizes) and can provide the capability needed to characterize proteins from as little as a single mammalian cell.

\section{Sensitivity}

FTICR is currently one of the most sensitive forms of MS (48), with dem- onstrated detection limits from 0.5 amol for peptides eluting from online capillary [50-75 $\mu \mathrm{m}$ inside diameter (i.d.)] LC (49) down to $30 \mathrm{zmol}$ for directly infused proteins (50). However, even higher sensitivity is desirable to enable comprehensive proteomic measurements for clinical applications. A key development in this area is the significant improvement in LC instrumentation that provides the enhanced sensitivity needed to analyze small samples. Because ESI sensitivity increases as the liquid flow rate to the ion source decreases, reducing the column i.d. is the most efficient way to improve sensitivity without sacrificing the separation quality. Thus, for example, the overall ESI efficiency with $15 \mu \mathrm{m}$ i.d. capillaries is expected to be approximately 100 -fold greater than with $150 \mu \mathrm{m}$ i.d. capillaries, simply based upon flow rate considerations. We have recently conducted analyses of complex peptide mixtures using capillary LC columns with i.d.s as small as $15 \mu \mathrm{m}$ and shown that the sensitivity continues to increase inversely as the mobile phase flow rates drop to as low as $20 \mathrm{~nL} / \mathrm{min}$ (51). In addition, we have demonstrated the advantages of using ultra-high pressure $(10,000 \mathrm{psi})$ reversed-phase capillary LC to achieve higher efficiency separations (e.g., with peak capacities of $>1000$ ) than with otherwise comparable separations at conventional pressure $(<3000$ psi and peak capacities of approximately 100) (51). An order of magnitude increase in the separation efficiency yielded an approximate 3 -fold boost in the number of detected peptides, thus significantly increasing the attainable proteome coverage $(12,52)$. One should note that achieving a higher efficiency separation by increasing the operating pressure currently requires significant expertise (to prepare the columns, reduce dead volumes, etc.) (53) and may be less practical for the greater proteomics community than commercially available multidimensional separations used in the shotgun approach. However, the improved sensitivity and throughput afforded by 1-D separation certainly rewards the effort required to adopt this technology. Furthermore, in our work, the nanoLC-FTICR proteomics platform has already demonstrated a rug- gedness comparable to conventional LC (54), but with greatly improved throughput, sensitivity, and dynamic range coverage of proteomics measurements (55).

As depicted in Figure 4, in addition to an FTICR, our nanoproteomics platform includes a nanoscale LC $(15 \mu \mathrm{m}$ i.d., $85 \mathrm{~cm}$ length column packed with $3 \mu \mathrm{m} \mathrm{C18}$ particles operating at 10,000 psi, with flow rates of approximately $20 \mathrm{~nL} / \mathrm{min}$ ) and a novel solid-phase $\mathrm{mi}-$ croextraction (microSPE) front end for fast introduction of larger sample volumes. The microSPE precolumn typically allows $10 \mu \mathrm{L}$ sample volumes to be loaded in less than 2 min with $<5 \%$ sample loss and is important because it facilitates future automation of the methodology. (This online microSPEnanoLC system has been described in more detail in Reference 57.)

To assess the sensitivity attainable with our nanoLC-FTICR platform, we analyzed both the prokaryote $D$. radiodurans and the protein bovine serum albumin (BSA). Using the AMT tag approach, we were able to identify 5 BSA peptides from $75 \mathrm{zmol}$ (approximately 45,000 molecules) of BSA tryptic digest and estimate a peptide detection limit at $10 \mathrm{zmol}$ (approximately 6000 molecules) (56). Note, however, that the analysis of such small samples would likely be impractical due to the losses to the chromatographic and other surfaces in the absence of a carrier species.

In a single 3-h nanoLC-FTICR analysis of a 2.5-ng total $D$. radiodurans protein sample, 436 proteins were identified (or approximately $14 \%$ of the entire predicted proteome), roughly equivalent to that achieved previously using $150 \mu \mathrm{m}$ i.d. capillary LC-FTICR with $1 \mu \mathrm{g}$ sample quantities. The AMT tag enabled nanoLC-FTICR analysis of a 5-pg D. radiodurans sample, yielding 53 proteins through the identification (and quantitation) of ${ }^{14} \mathrm{~N} /{ }^{15} \mathrm{~N}$ peptide pairs, while the experiment with only $0.5 \mathrm{pg}$ sample still successfully identified the most abundant proteins in the cell (such as elongation factors, ribosomal proteins, and proteins associated with the S-layer). Therefore, assuming that a typical mammalian cell contains approximately $50 \mathrm{pg}$ of protein (58), with an average of approximately 200 
zmol per protein, the current state-ofthe-art nanoLC-FTICR methodology should enable the analysis of a single cell's protein content.

The overall 0.5 ppm (i.e., MMA $\times$ TMA) 2-D specificity has proven to be sufficient to characterize most microbial proteomes. Obviously, the overall mass and time specificity needed to differentiate peptides, and thus identify proteins with good confidence, depends on the proteome complexity. In silico digestion of approximately 50,000 human proteins (http://www. ensembl.org) yields approximately 20 -fold more tryptic peptides than $D$. radiodurans (not taking modified proteins into account), a level that appears to be tractable with 1 ppm MMA and 0.01 TMA without resorting to a second dimension of separation. However, the actual size and complexity of the human proteome (or any proteome for that matter) are unknown. Despite the knowledge of the sequenced genome, the number of potential proteins, including all (often transient) isoforms, is undefined (although the order of magnitudes are higher than the number of genes). In fact, due to the genetic events (e.g., alternative splicing) and PTMs, the number of protein isoforms can become enormous, as exemplified by the single gene unit in Drosophila that might give rise to $>38,000$ protein products (59). Recent evidence of post-translational protein splicing in vertebrates shows that the alternatives for converting genes into proteins are even greater than initially thought, thus further increasing the complexity of the proteome (60). Encouragingly, the high peak capacities and the accurate peptide elution order reproducibility of the initial nanoLC studies suggest that $<0.005$ TMA should be achievable, a level that would provide sufficient levels of confidence for protein identifications in studies of mammalian cells. Similarly, higher levels of MMA $(<0.25 \mathrm{ppm})$

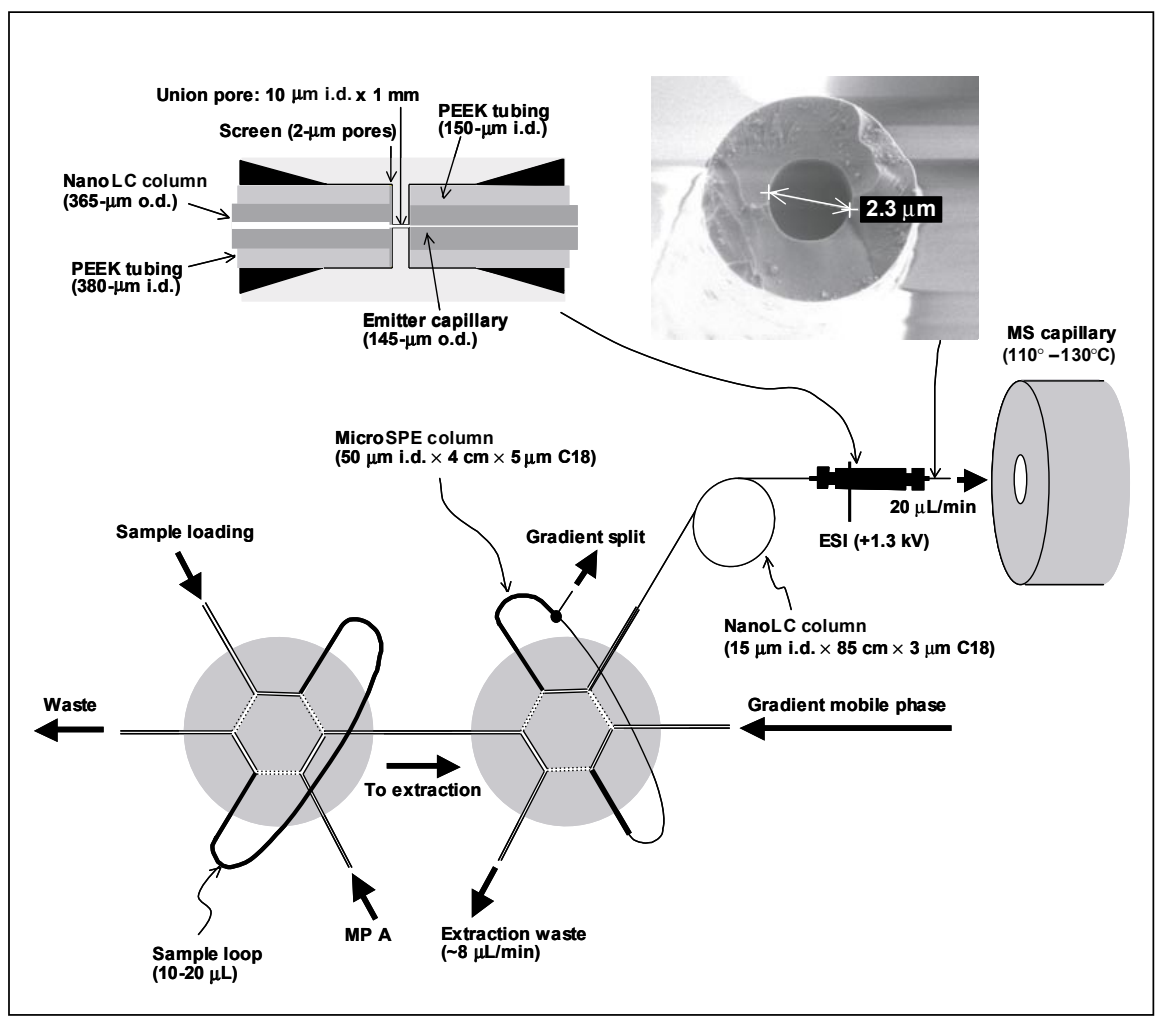

Figure 4. Diagram of the nanoLC system. The system includes a microextraction (microSPE) device for fast sample loading, a high-efficiency nanoLC for the separation, and a replaceable nanoESI source for sensitive ionization of the separated components. The SPE sample loading device and gradient nanoLC separations use automatic switching valves at 10,000 psi. The replaceable emitter (inset) was coupled through a stainless steel union where the high voltage was applied for nanoESI. Reproduced with permission from Reference 57. ESI, electrospray ionization; MS, mass spectrometry; i.d., inside diameter; MP A, mobile phase A. 
appear feasible using FTICR (61-63). Although routinely achieving these improved levels of performance will require more work, such effort is clearly warranted due to the large increase in throughput and sensitivity as well as instrumental robustness associated with a single dimension separation compared to multidimensional separations.

As an initial example of an application of the nanoproteomic measurement method to mammalian samples, we analyzed global cell lysates from the human mammary epithelial breast cell line MCF7 at low microgram levels. Two approximately $1 \mu \mathrm{g}$ (5000 cells) batches of MCF7 proteins were labeled with solid-phase isotope-coded affinity tags (SPICATs) to probe our ability to effectively process and analyze small clinically relevant samples. The nanoLC-FTICR analysis of a 25-ng sample derived from this preparation yielded $>1000$ SPICAT-labeled peptide pairs, dramatically exceeding any previous report in both sensitivity and Cys-containing peptide coverage. More than 5000 labeled Cys-containing peptide pairs were observed when a larger amount of protein (e.g., $50 \mu \mathrm{g}$ of total MCF7 protein) was used for (each) SPI-
CAT labeling and analyzed by FTICR coupled with a $50-\mu \mathrm{m}$ i.d. capillary LC column. As might be expected, the number of detected species significantly increases if one further eliminates the stable-isotope labeling step. For example, performing a conventional LC-FTICR analysis of ${ }^{14} \mathrm{~N} /{ }^{15} \mathrm{~N}$ metabolically labeled, tryptically digested MCF7 proteins $\left[{ }^{15} \mathrm{~N}\right.$-labeled $\mathrm{MCF}_{\text {neo }}$ cells vs. unlabeled (i.e., ${ }^{14} \mathrm{~N}$-labeled $\mathrm{MCF}_{\text {clone }}$ ${ }_{18}$ cells)] produced a data set containing $>10,000{ }^{14} \mathrm{~N} /{ }^{15} \mathrm{~N}$ labeled peptide pairs. Figure 5 shows a 2-D mass-versus-time display generated for the analyzed $5 \mu \mathrm{g}$ sample in comparison with a 2-D display generated for the analyzed $50 \mathrm{ng}$ sample, with insets showing the isotopic distributions for two representative peptide pairs. This figure illustrates the high sensitivity achievable using the nanoLC-FTICR proteomics platform, which allows a comparable number of detected ${ }^{14} \mathrm{~N} /{ }^{15} \mathrm{~N}$ peptide pairs as the analysis employing a $150-\mu \mathrm{m}$ i.d. capillary and two orders of magnitude larger sample loading.

\section{Dynamic Range}

The large variation in the relative

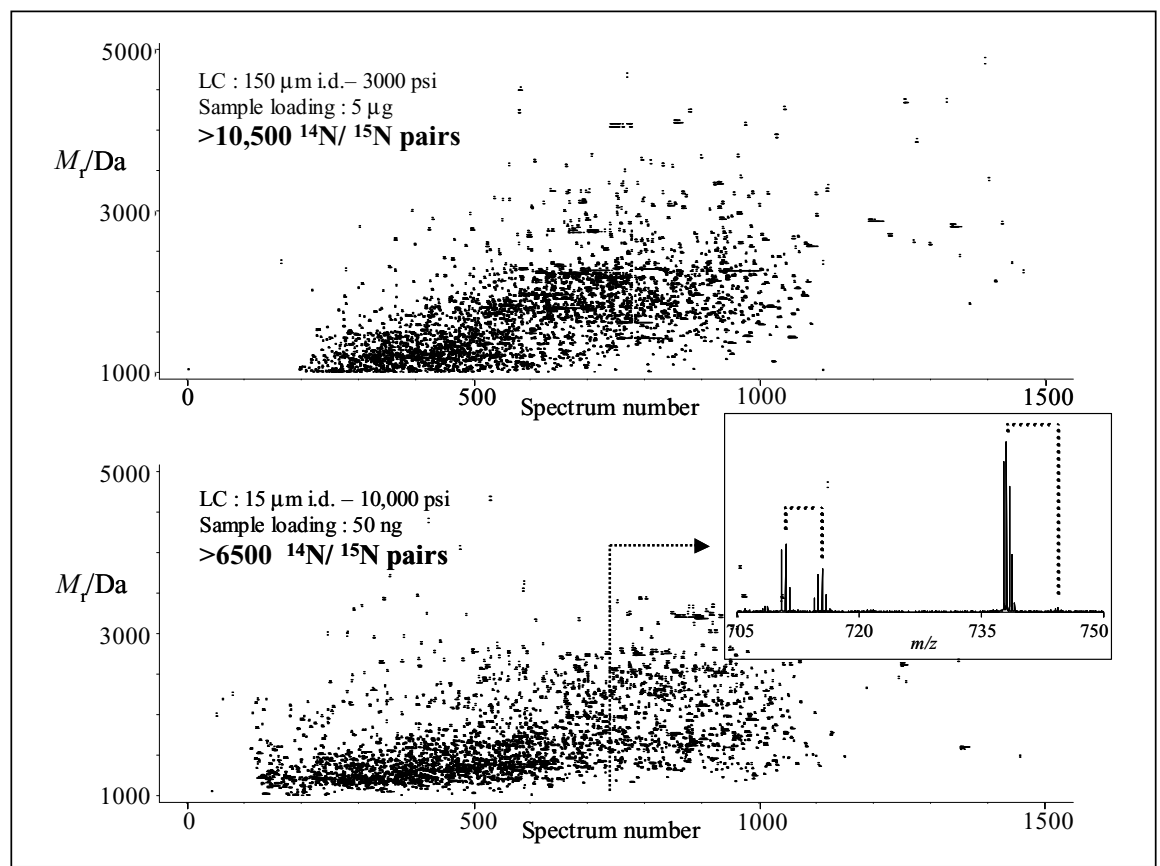

Figure 5. A two-dimensional (2-D) display from the nanoLC-Fourier transform ion cyclotron resonance (FTICR) analysis of $5 \mu \mathrm{g}$ from ${ }^{14} \mathrm{~N} /{ }^{15} \mathrm{~N}$ metabolically labeled tryptically digested proteome sample analyzed using a 150- $\mu \mathrm{m}$ i.d. column (top) and $50 \mathrm{ng}$ of the same sample loaded on a $15-\mu \mathrm{m}$ i.d. column (bottom). Insets show the isotopic distributions for two representative peptide pairs. i.d., inside diameter; $m / z$, mass-to-charge ratio. $M_{\mathrm{r}}$, molecular mass; $L C$, liquid chromatography. abundances of proteins in biological systems (potentially greater than $10^{10}$ in human serum) presents a major challenge for proteomics. The requirement to detect low-abundance (but often biologically important) species masked by the presence of highly abundant species puts a severe strain on the dynamic range (i.e., the ratio of the most abundant to the lowest abundant species detectable in a single analysis) of the experimental approaches used.

To determine the range of relative protein abundances measurable by nanoLC-FTICR for complex proteomics sample, we examined a mixture of $75 \mathrm{zmol}$ of BSA tryptic digest, 75 fmol of cytochrome $c$ tryptic digest, and $5 \mathrm{ng}$ of ${ }^{14} \mathrm{~N} /{ }^{15} \mathrm{~N}$ metabolically labeled $D$. radiodurans proteome tryptic digest. Observation of the tryptic peptides from all three sources (i.e., BSA, cytochrome $c$, and $D$. radiodurans) sets the dynamic range of the LC-FTICR measurement at $10^{6}(55)$.

In many cases, the presence of highly abundant peptides can preclude detection of low-abundance peptides in the same spectrum. This issue can be effectively addressed using dynamic range enhancement applied to mass spectrometry (DREAMS), a recently developed approach that involves the use of data-directed ejection of the most abundant ions prior to ion accumulation in a 2-D ion trap external to the ion cyclotron resonance (ICR) trap $(64,65)$. Due to the elimination of the major ions in this way, which would otherwise result in the rapid filling of the external 2-D quadrupole ion trap and, consequently, the ICR trap, one can then selectively accumulate lower abundance species for a significantly extended period of time and achieve both greater sensitivity and extended dynamic range.

We have demonstrated the DREAMS approach in the analysis of tryptic peptides using ${ }^{14} \mathrm{~N} /{ }^{15} \mathrm{~N}$ labeled $D$. radiodurans and mouse B16 melanoma proteins and showed that the number of detected peptide pairs suitable for use in quantitative analyses is significantly increased due to the enhanced dynamic range of the measurements. As an example, a combined total of 17,813 unambiguous and unique ${ }^{14} \mathrm{~N} /{ }^{15} \mathrm{~N}$ peptide pairs with an average 
abundance ratio of 1.035 and standard deviation of 0.29 was detected in a single DREAMS LC-FTICR analysis of mouse B16 melanoma proteins. Importantly, by using DREAMS, the number of peptides for which quantitative information could be obtained (from the relative abundances of peptide pairs) was increased by $80 \%$. In order to address the question of whether such a huge increase in the number of observed peptides translates into markedly better proteome coverage, we have analyzed a mixture of ${ }^{14} \mathrm{~N} /{ }^{15} \mathrm{~N}$-labeled D. radiodurans cells. The overall proteome coverage achieved in these experiments can be compared with recent work by Washburn et al. $(66,67)$, using MudPIT, which reported an average of 869 yeast proteins (approximately 14\% of the predicted larger set of yeast proteins) observed in three analyses of $1: 1$, $5: 1$, and $10: 1$ mixtures of yeast grown in ${ }^{14} \mathrm{~N}$ - and ${ }^{15} \mathrm{~N}$-enriched minimal media. Using the AMT tag approach, we observed and quantified $1244 \mathrm{D}$. radiodurans proteins (approximately $40 \%$ of the predicted $D$. radiodurans proteome) as peptide pairs in a single DREAMS LC-FTICR analysis. Furthermore, DREAMS and nanoLC-FTI$\mathrm{CR}$ technologies were recently used in combination with SPICAT to achieve the highest proteome coverage $(>2000$ Cys-containing peptide pairs) from the smallest amount of the starting protein $(10,000$ mammalian cells or $<2 \mu \mathrm{g})$. In comparison, the best previously published data reported approximately 80 and 700 Cys-containing pairs starting with $10 \mu \mathrm{g}$ and $2.5 \mathrm{mg}$ of total protein, respectively $(35,68-70)$.

\section{Comparison with Conventional Technology}

The 75-zmol (FTICR) and 7-amol (ion trap MS/MS) sensitivities required sample sizes from $D$. radiodurans of 0.5 and $50 \mathrm{pg}$, respectively, in order to identify the more abundant proteins that accounted for $0.2 \%$ to $2.0 \%$ (w/w) of the total protein content (57). The dynamic range measured for $2.5 \mathrm{ng}$ (total protein) sample was approximately $10^{6}$ using FTICR, in comparison with approximately $10^{4}$ for ion trap MS/MS (55). The average proteome measurement throughput of approximately 50 pro- teins/h using MS/MS during reversedphase LC separation presently requires $3 \mathrm{~h} /$ sample. As expected, the AMT tag approach significantly increased throughput (i.e., to approximately 300 proteins/h) due to the fact that $>100$ (and up to $>10,000)$ species can be detected in a single spectrum $(11,71)$.

Figure 6 shows the proteome coverage obtained for $0.5 \mathrm{pg}$ to $10 \mathrm{ng}$ total protein using nanoLC with both FTICR and ion trap MS/MS. The results show that the number of proteins identified using FTICR increases little for samples $>2.5 \mathrm{ng}$, suggesting that most proteins were identified at this level. The corresponding nanoLC-ion trap MS/MS analyses produced a proteome coverage of $4.5 \%$ from $10 \mathrm{ng}$ proteins, due to both the decreased sensitivity of the ion trap and the limitations imposed by the number of tandem MS spectra that can be obtained during the separation. Increasing the protein amounts used, optimizing the nanoLC gradient profile, and fractionating proteins (i.e., using multiple fraction analyses or 2-D separations) prior to LC-MS/MS measurement enable improved coverage of lower abundance peptides by tandem MS but correspondingly decrease sensitivity and throughput.

\section{Achieving Quantitation}

To fully realize the potential of the AMT tag approach requires the exploitation of its high-throughput capability to determine protein abundances in a wide range of conditions. Figure 7A illustrates the generation of an AMT tag for the Schewanella oneidensis translation elongation factor Tu (tufB). A tryptic digest of $S$. oneidensis proteins was previously analyzed repeatedly by LC MS/MS using a conventional ion trap mass spectrometer to identify a large number of peptides. One of the peptides selected for dissociation was identified as ELLSEYDFPGDDLPVIQGSALK from the protein tufB and was considered as a potential mass and time (PMT) tag. The theoretical mass of this peptide was then calculated $(2,405.2001 \mathrm{u})$ based on the elemental composition of the identified sequence and its normalized LC retention time recorded (0.502). The sample was then analyzed by capillary LC-FTICR in the 
MS-only mode using identical LC separation conditions. The data set is represented here as a 2-D display, where each spot represents one detected isotopic distribution, its coordinates being retention time and molecular mass. In this particular acquisition, a peptide was detected with an observed mass of $2405.1977 \mathrm{u}$ and a normalized retention time of 0.504 that closely agree with the calculated mass of our PMT tag (within $1 \mathrm{ppm}$ ) and its recorded retention time (within 5\%). As this peptide falls within the criteria established for generation of an AMT tag, it is now considered to be confidently assigned as an AMT tag for tufB. In subsequent analyses of the $S$. oneidensis proteome by capillary LC-FTICR, a species detected at the same mass and retention time will immediately be identified as the AMT tag from tufB without the need to resort to MS/MS as shown in Figure 7B. In this figure, two cell populations were grown in isotopically distinct media under aerobic (natural isotopic abundance; i.e., ${ }^{14} \mathrm{~N}$ medium) and suboxic conditions $\left({ }^{15} \mathrm{~N}\right.$-enriched medium). The resulting cell lysates were mixed and digested using trypsin. The 2-D mass and time tag display presents a portion of the LC-FTICR data set, and the inset shows a peptide pair identified as the previously confirmed AMT from tufB. The disparity in mass between the two versions of the peptide corresponds to 24 times the difference between ${ }^{15} \mathrm{~N}$ and ${ }^{14} \mathrm{~N}$, which confirms our AMT tag identification (ELLSEYDFPGDDLPVIQGSALK indeed contains $24 \mathrm{~N}$ atoms). The abundance ratio of those two peptides is close to one, indicating that the protein tufB is expressed in similar amounts under aerobic and suboxic conditions.

Thus, once a protein has been identified using AMT tags, it can be directly followed in a fashion analogous to an identified 2-DE spot. However, the "spots" can be defined with much greater accuracy using relative LC retention times and accurate mass measurements, yielding greater specificity compared to 2-DE. Additionally, measurements can be made with greater sensitivity and dynamic range. Finally, high-throughput studies of proteomes become possible, without the need to reestablish a peptide's identity in every analysis using MS/MS.

\section{Targeted Comparative Proteomics}

In some cases, the use of MS/MS for peptide identification and the generation of an AMT tag database is not practical, such as when a sample is rare or precious (e.g., biopsies) or when one seeks only to monitor previously identified biomarkers. In these cases, the use of a targeted MS/MS approach is more attractive for identifying peptides that show significant differences between culture conditions and/or dis-

eased states. Targeted MS/MS is accomplished using isotopically labeled samples. The sample is first analyzed in MS-only mode on an LC-FTICR system, and the abundance ratios of the detected peptide pairs are calculated. The $m / z$ values and elution times of the interesting pairs (i.e., pairs showing differential abundances between the two isotopically labeled versions of the peptide) are consolidated into an "attention list." Next, an LC-MS/MS experiment is conducted in which the attention list directs the MS/MS acquisition to specifically target the species having the greatest potential for being markers of a specific state (e.g., disease markers). This approach was initially demonstrated on a mixture of SPICATlabeled protein standards. The strategy was shown to circumvent problems associated with the elution differences of two isotopically distinct peptides and allowed confident identification of the protein with a 6:1 isotopic ratio in our mixture. The method was also applied to $S$. oneidensis for cells grown in isotopically distinct media $\left({ }^{14} \mathrm{~N} /{ }^{15} \mathrm{~N}\right)$ under aerobic and suboxic conditions. Using a targeted MS/MS approach, several proteins known to be differentially expressed in suboxic versus aerobic conditions in S. oneidensis were identified (e.g., fumarate reductase; C. Masselon, L. Paša-Tolić, G.A. Anderson, B. Bogdanov, A.N. Vilkov, Y. Shen, R. Zhou, M.S. Lipton, D.G. Camp, and R.D. Smith, manuscript submitted).

\section{CONCLUSION AND PERSPECTIVES}

As researchers extend proteomic technology to study more complex systems, there is an increasing need for tools that can better identify and quantify the proteome of an organism. Greater confidence in peptide assignment is now being accomplished using ANNs that evaluate whether a putative peptide is predicted to have the elution time as observed experimentally. Training sets of $>5100$ peptides (with known amino acid sequences) from approximately 700 LC-MS/MS analyses were initially used to establish the ANN prediction algorithm. Our model can predict the elution times of peptides (with

Figure 6. Number of identified proteins and corresponding proteome coverage as a function of the sample amount loaded on the column obtained using nanoLC- FTICR and ion trap tandem MS analyses of ${ }^{14} \mathrm{~N} /{ }^{15} \mathrm{~N}$-labeled Deinoccocus radiodurans peptides. All LC-FTICR identifications were based on AMT tags while ion trap tandem MS identifications used conventional approaches. Reproduced with permission from Reference 57. The error bars represent the variance estimated from replicate analyses of some samples. FTICR, Fourier transform ion cyclotron resonance; ORFs, open reading frames; LC, liquid chromatography; MS, mass spectrometry; AMT, accurate mass and time. 
up to 54 amino acids) within $3 \%$ (interquartile range) of their observed times (44). Furthermore, elution times of $>95 \%$ of all peptides under study can be predicted with an absolute error of $<10 \%$ (44). Because a particular signal can potentially match numerous peptides within a large database, we use ANN to compare the actual normalized elution time (NET) value for each putative amino acid string (peptide) to its predicted value (as establish from a linear NET equation) and retain only those identifications that fall within an absolute error of $10 \%$. Therefore, the use of ANN provides greater confidence in AMT tag assignments and is a useful filter for removing false identifications. For simpler mixtures, the AMT tag approach could ultimately use only accurate mass and predicted elution times to identify peptides without MS/ MS information.

To better characterize proteomes, we are using the AMT tag strategy to target interesting peptide signals for automated LC-FTICR-MS/MS analysis. Invariably, the analysis of two different cellular states will yield signals that can be attributed to changes in protein PTMs or differences in protein abundance levels. We are thus developing an AMT tag approach that can identify the presence of newly detected peptides and create an attention list of ions that require FTICR-MS/MS analysis for further on-the-fly characterization. This type of approach is well suited for use with clinical samples of limited size because the datadirected MS/MS capability could selectively characterize detected peptide ions that do not appear on a previously established list from control samples. When further coupled with DREAMS technology, data-directed LC-FTICRMS/MS analysis could potentially reveal low-abundance proteins crucial for cellular response to certain perturbations (e.g., low-abundance transcription factors known to be associated with human cancers).

Efforts are also underway to extend the AMT tag approach to intact protein analysis to better identify the mature form(s) of proteins in vivo. The goal is to take advantage of the valuable information on protein size and modifications as generated by both the top-down and bottom-up approaches. In previous work, we applied stable-isotope labeling for quantitative proteomics at the intact protein level and demonstrated the ability to make precise measurements of differences in the abundances of many proteins using capillary isoelectric focusing coupled with FTICR for intact Escherichia coli proteins (72). We are also engaged in efforts to exploit the extremely high ionization efficiency and concentration dependence afforded by the nanoflow ESI process to estimate the absolute abundance (as opposed to relative abundance) of proteins in cells or tissues as a function of cellular condition. In many cases, individual protein abundance may vary over a large dynamic range depending on the functional classification and localization of the protein within the organism. The relative abundance levels between various proteins may provide valuable information as to the active utilization of biochemical networks and metabolic pathways. Our nanoproteomic platform is ideally suited for this type of work because we use a very small capillary LC column (15 $\mu \mathrm{m}$ i.d.) and the resulting low flow rate (approximately $20 \mathrm{~nL} / \mathrm{min}$ ) to optimize ionization efficiency. The added advantage of the combined nanoproteomics platform and AMT tag approach is that we are able to obtain large ion signal counts per peptide and thereby achieve highquality quantitative data on protein abundance. ANN-type approaches are also being investigated to correct for the variability in electrospray response typically detected among structurally diverse peptides to obtain the more accurate quantitation.

The ability to comprehensively identify and quantify protein abundances in complex biological systems is considered essential for revealing the expression machinery that is employed by an organism in response to stimuli and to understand disease. The field of pro- 
A

AMT \#1146 [ORF \#02142]

B

translation elongation factor $\mathrm{Tu}$ (tufB)

\section{ELLSEYDFPGDDLPVIQGSALK}

Theor. $M_{\mathrm{r}}=2,405.2001 \mathrm{Da}$

$\mathrm{NET}=0.502$
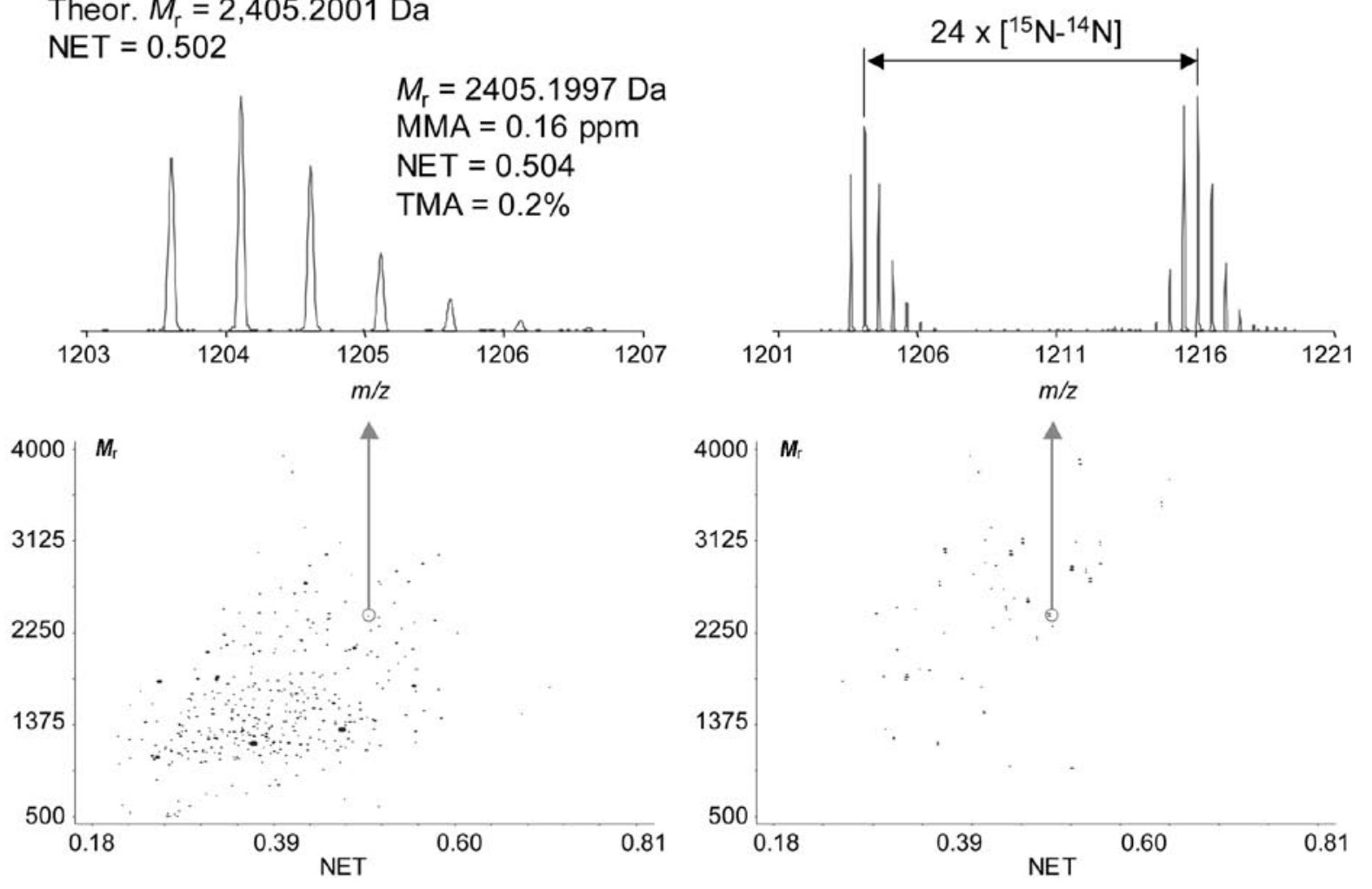

Figure 7. Two-dimensional (2-D) display for capillary LC-FTICR analysis of Schewanella oneidensis proteome (B) shows detected species at their normalized elution times (NET) and molecular masses $\left(\boldsymbol{M}_{\mathbf{r}}\right)$. Peptide shown in panel A (ELLSEYDFPGDDLPVIQGSALK) for translation elongation factor Tu. (D) The 2-D display for a mixture of two $\mathrm{S}$. oneidensis cultures under aerobic $\left({ }^{14} \mathrm{~N}\right.$-labeled) and suboxic $\left({ }^{15} \mathrm{~N}\right.$-labeled) conditions is also shown. $(\mathrm{C}) \mathrm{The}$ light member of a peptide pair was identified as the same AMT tag (by $M_{\mathrm{r}}$ and NET) from translation elongation factor Tu. The abundance ratio of the peptide pair indicates that this protein was not significantly altered in suboxic versus aerobic conditions. FTICR, Fourier transform ion cyclotron resonance; LC, liquid chromatography; AMT, accurate mass and time; MMA, mass measurement accuracy; TMA, time measurement accuracy; $\mathrm{m} / \mathrm{z}$, mass-to-charge ratio.

teomics has made rapid progress in recent years toward fulfilling this requirement, but better approaches are needed before proteomics can realize its full potential. The capillary LC-FTICR platform and AMT tag approach can produce extremely high-resolution 1-D separations (peak capacities $>1000$ ) with simultaneous large dynamic range detection $\left(>10^{6}\right)$ and $\mathrm{zmol}$ $\left(10^{-21} \mathrm{~mol}\right)$ sensitivity, while increasing throughput and providing high mass measurement accuracies for confident protein identification and quantitation, without the need for repetitive MS/MS analyses. The approach has now been successfully applied to the study of $>20$ different organisms from all three domains of life, including mammalian systems. The current state of the art of the AMT tag strategy allows smaller amounts of sample to be analyzed in an automated high-throughput fashion and protein identifications to be made to greater depths, while quantifications can by made with better accuracy, precision, and greater confidence.

Future studies will continue to seek improved approaches to better identify and quantify protein PTMs and process very small amounts of sample. Bioinformatic tools will also be refined to aid researchers in visualizing and interpreting proteomic data so that results can be more easily understood and communicated. Further, collaborative efforts are underway to integrate AMT tag data results with data obtained from complimentary "omic" studies to facilitate our collective pursuit to understand the inner workings of the cell.

\section{ACKNOWLEDGMENTS}

L. Paša-Tolic', C. Masselon, and R.C. Barry contributed equally to this work. The authors are grateful to all past and present members of the MS group in the Environmental Molecular Sciences Laboratory, Pacific Northwest National Laboratory involved in this work. We also thank Dr. Maria Pallavicini for metabolically labeled MCF7 cells and Ms. Penny Colton for critically reviewing the manuscript and providing helpful suggestions. Portions 
of this research were supported by the U.S. Department of Energy (DOE) Office of Biological and Environmental Research, the National Cancer Institute under grant nos. CA81654 and CA86340, and the National Center for Research Resources (grant no. RR18522) to R.D.S. Pacific Northwest National Laboratory is operated by Battelle for the DOE under contract no. DE-AC06-76RLO 1830.

\section{COMPETING INTERESTS STATEMENT}

The authors declare no conflicts of interest.

\section{REFERENCES}

1.Baak, J.P., F.R. Path, M.A. Hermsen, G. Meijer, J. Schmidt, and E.A. Janssen. 2003. Genomics and proteomics in cancer. Eur. J. Cancer 39:1199-1215.

2.Anderson, L. and J. Seilhamer. 1997. A comparison of selected mRNA and protein abundances in human liver. Electrophoresis 18:533-537.

3.Gygi, S.P., Y. Rochon, B.R. Franza, and R. Aebersold. 1999. Correlation between protein and mRNA abundance in yeast. Mol. Cell Biol. 19:1720-1730.

4.Hochstrasser, D.F. 1998. Proteome in perspective. Clin. Chem. Lab. Med. 36:825-836.

5.Wang, Y., C.L. Liu, J.D. Storey, R.J. Tibshirani, D. Herschlag, and P.O. Brown. 2002. Precision and functional specificity in mRNA decay. Proc. Natl. Acad. Sci. USA 99:58605865.

6.Karas, M., U. Bahr, and U. Giessmann. 1991. Matrix-assisted laser desorption ionization mass-spectrometry. Mass Spectrom. Rev. 10:335-357.

7.Henzel, W.J., C. Watanabe, and J.T. Stults. 2003. Protein identification: the origins of peptide mass fingerprinting. J. Am. Soc. Mass Spectrom. 14:931-942.

8.Covey, T.R., E.C. Huang, and J.D. Henion. 1991. Structural characterization of protein tryptic peptides via liquid-chromatography mass-spectrometry and collision-induced dissociation of their doubly charged molecularions. Anal. Chem. 63:1193-1200.

9.Yates, J.R. 1998. Mass spectrometry and the age of the proteome. J. Mass Spectrom. 33:119.

10.Aebersold, R. and M. Mann. 2003. Mass spectrometry-based proteomics. Nature 422:198-207.

11.Bruce, J.E., G.A. Anderson, J. Wen, R. Harkewicz, and R.D. Smith. 1999. High mass measurement accuracy for $100 \%$ sequence coverage of enzymatically digested bovine serum albumin from an ESI-FTICR mass spectrum. Anal. Chem. 71:2595-2599.

12.Shen, Y., R. Zhao, M.E. Belov, T.P. Con- rads, G.A. Anderson, K. Tang, L. PašaTolić, T.D. Veenstra, et al. 2001. Packed capillary reversed-phase liquid chromatography with high-performance electrospray ionization Fourier transform ion cyclotron resonance mass spectrometry for proteomics. Anal. Chem. 73:1766-1775.

13.Page, J.S., C.D. Masselon, and R.D. Smith. 2004. FTICR mass spectrometry for qualitative and quantitative bioanalyses. Curr. Opin. Biotechnol. 15:3-11.

14.McDonald, W.H. and J.R. Yates, 3rd. 2002. Shotgun proteomics and biomarker discovery. Dis. Markers 18:99-105.

15.McDonald, W.H. and J.R. Yates, 3rd. 2000. Proteomic tools for cell biology. Traffic 1:747-754

16.Patterson, S.D. and R. Abersold. 1995. Mass spectrometric approaches for the identification of gel-separated proteins. Electrophoresis 16:1791-1814

17.Aebersold, R. and J. Leavitt. 1990. Sequence analysis of proteins separated by polyacrylamide gel electrophoresis: towards an integrated protein database. Electrophoresis 11:517-527.

18.Barry, R.C., B.L. Alsaker, J.F. RobisonCox, and E.A. Dratz. 2003. Quantitative evaluation of sample application methods for semipreparative separations of basic proteins by two-dimensional gel electrophoresis. Electrophoresis 24:3390-3404.

19.Gygi, S.P., G.L. Corthals, Y. Zhang, Y. Rochon, and R. Aebersold. 2000. Evaluation of two-dimensional gel electrophoresis-based proteome analysis technology. Proc. Natl. Acad. Sci. USA 97:9390-9395.

20.Liu, H., D. Lin, and J.R. Yates, 3rd. 2002. Multidimensional separations for protein/ peptide analysis in the post-genomic era. BioTechniques 32:898-902.

21.McDonald, W.H. and J.R. Yates, 3rd. 2003. Shotgun proteomics: integrating technologies to answer biological questions. Curr. Opin. Mol. Ther. 5:302-309.

22.Goodlett, D.R. and E.C. Yi. 2002. Proteomics without polyacrylamide: qualitative and quantitative uses of tandem mass spectrometry in proteome analysis. Funct. Integr. Genomics 2:138-153.

23.Ducret, A., I. Vanoostveen, J.K. Eng, J.R. Yates, and R. Aebersold. 1998. High throughput protein characterization by automated reverse-phase chromatography electrospray tandem mass spectrometry. Protein Sci. 7:706-719.

24.Link, A.J., J. Eng, D.M. Schieltz, E. Carmack, G.J. Mize, D.R. Morris, B.M. Garvik, and J.R. Yates. 1999. Direct analysis of protein complexes using mass spectrometry. Nature Biotechnol. 17:676-682.

25.Wolters, D.A., M.P. Washburn, and J.R. Yates. 2001. An automated multidimensional protein identification technology for shotgun proteomics. Anal. Chem. 73:5683-5690.

26.Paša-Tolić, L., R. Harkewicz, G.A. Anderson, N. Tolić, Y. Shen, R. Zhao, B. Thrall, C. Masselon, et al. 2002. Increased proteome coverage based upon high performance separations and DREAMS FTICR mass spectrometry. J. Am. Soc. Mass Spectrom. 13:954-963.

27.Conrads, T.P., G.A. Anderson, T.D. Veen- stra, L. Paša-Tolić, and R.D. Smith. 2000. Utility of accurate mass tags for proteomewide protein identification. Anal. Chem. 72:3349-3354

28.Tao, W.A. and R. Aebersold. 2003. Advances in quantitative proteomics via stable isotope tagging and mass spectrometry. Curr. Opin. Biotechnol. 14:110-118.

29.Zhang, R., C.S. Sioma, S. Wang, and F.E. Regnier. 2001. Fractionation of isotopically labeled peptides in quantitative proteomics. Anal. Chem. 73:5142-5149.

30.Ferguson, P.L. and R.D. Smith. 2003. Proteome analysis by mass spectrometry. Annu. Rev. Biophys. Biomol. Struct. 32:399-424.

31.Yao, X.D., A. Freas, J. Ramirez, P.A. Demirev, and C. Fenselau. 2001. Proteolytic 180 labeling for comparative proteomics: model studies with two serotypes of adenovirus. Anal. Chem. 73:2836-2842.

32.Oda, Y., K. Huang, F.R. Cross, D. Cowburn, and B.T. Chait. 1999. Accurate quantitation of protein expression and site-specific phosphorylation. Proc. Natl. Acad. Sci. USA 96:6591-6596.

33.Paša-Tolić, L., P.K. Jensen, G.A. Anderson, M.S. Lipton, K.K. Peden, S. Martinovic, N. Tolić, J.E. Bruce, et al. 1999. High throughput proteome-wide precision measurements of protein expression using mass spectrometry. J. Amer. Chem. Soc. 121:7949-7950.

34.Gygi, S.P., B. Rist, S.A. Gerber, F. Turecek, M.H. Gelb, and R. Aebersold. 1999. Quantitative analysis of complex protein mixtures using isotope-coded affinity tags. Nature Biotechnology 17:994-999.

35.Zhou, H.L., J.A. Ranish, J.D. Watts, and R. Aebersold. 2002. Quantitative proteome analysis by solid-phase isotope tagging and mass spectrometry. Nat. Biotechnol. 19:512-515.

36.Qian, W.J., M.B. Goshe, D.G. Camp, 2nd, L.R. Yu, K. Tang, and R.D. Smith. 2003 Phosphoprotein isotope-coded solid-phase tag approach for enrichment and quantitative analysis of phosphopeptides from complex mixtures. Anal. Chem. 75:5441-5450.

37.Goshe, M., T. Conrads, E. Panisko, N. Angell, T. Veenstra, and R. Smith. 2001. Phosphoprotein isotope-coded affinity tag approach for isolating and quantitating phosphopeptides in proteome-wide analyses. Anal. Chem. 73:2578-2586.

38.Goshe, M.B. and R.D. Smith. 2003. Stable isotope-coded proteomic mass spectrometry. Curr. Opin. Biotechnol. 14:101-109.

39.Gerber, S.A., J. Rush, O. Stemman, M.W. Kirschner, and S.P. Gygi. 2003. Absolute quantification of proteins and phosphoproteins from cell lysates by tandem MS. Proc. Natl. Acad. Sci. USA 100:6940-6945.

40.Eng, J.K., A.L. McCormack, and J.R. Yates. 1994. An approach to correlate tandem mass spectral data of peptides with amino acid sequences in a protein database. J. Am. Soc. Mass Spectrom. 5:976-989.

41.MacCoss, M.J. and J.R. Yates, 3rd. 2001 Proteomics: analytical tools and techniques. Curr. Opin. Clin. Nutr. Metab. Care 4:369375.

42.MacCoss, M.J., C.C. Wu, H. Liu, R. Sadygov, and J.R. Yates, 3rd. 2003. A correlation algorithm for the automated quantitative anal- 
ysis of shotgun proteomics data. Anal. Chem. 75:6912-6921.

43.Yi, E.C., M. Marelli, H. Lee, S.O. Purvine, R. Aebersold, J.D. Aitchison, and D.R. Goodlett. 2002. Approaching complete peroxisome characterization by gas-phase fractionation. Electrophoresis 23:3205-3216.

44.Petritis, K., L.J. Kangas, P.L. Ferguson, G.A. Anderson, L. Paša-Tolić, M.S. Lipton, K.J. Auberry, E. Strittmatter, et al. 2003. Use of artificial neural networks for the prediction of peptide liquid chromatography elution times in proteome analyses. Anal. Chem. 75:1039-1048.

45.Lipton, M.S., L. Paša-Tolić, G.A. Anderson, D.J. Anderson, D.L. Auberry, J.R. Battista, M.J. Daly, J. Fredrickson, et al. 2002. Global analysis of the Deinococcus radiodurans proteome by using accurate mass tags. Proc. Natl. Acad. Sci. USA 99:11049-11054.

46.Belov, M.E., G.A. Anderson, M.A. Wingerd, H.R. Udseth, K. Tang, D.C. Prior, K.R. Swanson, M.A. Buschbach, et al. 2004. An automated high performance capillary liquid chromatography-Fourier transform ion cyclotron resonance mass spectrometer for highthroughput proteomics. J. Am. Soc. Mass Spectrom. 15:212-232.

47.Strittmatter, E.F., P.L. Ferguson, K. Tang, and R.D. Smith. 2003. Proteome analyses using accurate mass and elution time peptide tags with capillary LC time-of-flight mass spectrometry. J. Am. Soc. Mass Spectrom. 14:980-991.

48.Marshall, A.G., C.L. Hendrickson, and G.S. Jackson. 1998. Fourier transform ion cyclotron resonance mass spectrometry: a primer. Mass Spectrom. Rev. 17:1-35.

49.Martin, S.E., J. Shabanowitz, D.F. Hunt, and J.A. Marto. 2000. Subfemtomole MS and MS/MS peptide sequence analysis using nano-HPLC micro-ESI Fourier transform ion cyclotron resonance mass spectrometry. Anal. Chem. 72:4266-4274.

50.Belov, M.E., M.V. Gorshkov, H.R. Udseth, G.A. Anderson, and R.D. Smith. 2000. Zeptomole-sensitivity electrospray ionizationFourier transform ion cyclotron resonance mass spectrometry of proteins. Anal. Chem. 72:2271-2279.

51.Shen, Y., R. Zhao, S.J. Berger, G.A. Anderson, N. Rodriguez, and R.D. Smith. 2002. High-efficiency nanoscale liquid chromatography coupled on-line with mass spectrometry using nanoelectrospray ionization for proteomics. Anal. Chem. 74:4235-4249.

52.Shen, Y., N. Tolić, R. Zhao, L. Paša-Tolić, L. Li, S.J. Berger, R. Harkewicz, G.A. Anderson, et al. 2001. High-throughput proteomics using high efficiency multiple-capillary liquid chromatography with on-line high performance ESI FTICR mass spectrometry. Anal. Chem. 73:3011-3021.

53.Licklider, L.J., C.C. Thoreen, J. Peng, and S.P. Gygi. 2002. Automation of nanoscale microcapillary liquid chromatography-tandem mass spectrometry with a vented column. Anal. Chem. 74:3078-3083.

54.Shen, Y., J.M. Jacobs, D.G. Camp, 2nd, R. Fang, R.J. Moore, R.D. Smith, W. Xiao, R.W. Davis, et al. 2004. Ultra-high-efficiency strong cation exchange LC/RPLC/MS/MS for high dynamic range characterization of the human plasma proteome. Anal. Chem. 76:1134-1144.

55.Shen, Y., N. Tolić, C. Masselon, L. PašaTolić, D.G. Camp, 2nd, M.S. Lipton, G.A. Anderson, and R.D. Smith. 2004. Nanoscale proteomics. Anal. Bioanal. Chem. 378:10371045.

56.Shen, Y., R.J. Moore, R. Zhao, J. Blonder, D.L. Auberry, C. Masselon, L. Paša-Tolić, K.K. Hixson, et al. 2003. High-efficiency on-line SPE coupling to $15-150 \mathrm{~mm}$ i.d. column LC for proteomic analysis. Anal. Chem. 75:3596-3605.

57.Shen, Y., N. Tolić, C. Masselon, L. PašaTolić, D.G. Camp II, K.K. Hixson, R. Zhao, G.A. Anderson, et al. 2004. Ultrasensitive proteomics using high-efficiency on-line micro-SPE-NanoLC-NanoESI MS and MS/MS. Anal. Chem. 76:144-154.

58.Zhang, Z., S. Krylov, E.A. Arriaga, R. Polakowski, and N.J. Dovichi. 2000. Onedimensional protein analysis of an HT29 human colon adenocarcinoma cell. Anal. Chem. 72:318-322.

59.Black, D.L. 2000. Protein diversity from alternative splicing: a challenge for bioinformatics and post-genome biology. Cell 103:367-370.

60.Hanada, K., J.W. Yewdell, and J.C. Yang. 2004. Immune recognition of a human renal cancer antigen through post-translational protein splicing. Nature 427:252-256.

61.Masselon, C., A. Tolmachev, G. Anderson, R. Harkewicz, and R. Smith. 2002. Mass measurement errors caused by local frequency perturbations in FTICR mass spectrometry. J. Amer. Soc. Mass Spectrom. 13:99-106.

62.Tolmachev, A., C. Masselon, G. Anderson, H. Udseth, and R. Smith. 2002. Frequency shifts due to interface of resolved peaks in magnitude-mode Fourier-transform ion cyclotron resonance mass spectra. J. Am. Soc. Mass Spectrom. 13:387-401.

63.Savitski, M.M., I.A. Ivonin, M.L. Nielsen, R.A. Zubarev, Y.O. Tsybin, and P. Hakansson. 2004. Shifted-basis technique improves accuracy of peak position determination in Fourier transform mass spectrometry. J. Am. Soc. Mass Spectrom. 15:457-461.

64.Belov, M.E., G.A. Anderson, N.H. Angell, Y. Shen, N. Tolić, H.R. Udseth, and R.D. Smith. 2001. Dynamic range expansion applied to mass spectrometry based on datadependent selective ion ejection in capillary liquid chromatography Fourier transform ion cyclotron resonance for enhanced proteome characterization. Anal. Chem. 73:5052-5060.

65.Harkewicz, R., M.E. Belov, D.A. Anderson, L. Paša-Tolić, C.D. Masselon, D.C. Prior, H.R. Udseth, and R.D. Smith. 2002. ESI-FTICR mass spectrometry employing data-dependent external ion selection and accumulation. J. Amer. Soc. Mass Spectrom. 13:144-154.

66.Washburn, M.P., R. Ulaszek, C. Deciu, D. Schieltz, and J.R. Yates. 2002. Analysis of quantitative proteomic data generated via multidimensional protein identification technology. Anal. Chem. 74:1650-1657.

67.Washburn, M.P., D. Wolters, and J.R. Yates. 2001. Large-scale analysis of the yeast proteome by multidimensional protein identi- fication technology. Nat. Biotechnol. 19:242247

68.Guina, T., M. Wu, S.I. Miller, S.O. Purvine, E.C. Yi, J. Eng, D.R. Goodlett, R. Aebersold, et al. 2003. Proteomic analysis of Pseudomonas aeruginosa grown under magnesium limitation. J. Am. Soc. Mass Spectrom. 14:742-751.

69.Shiio, Y., S. Donohoe, E.C. Yi, D.R. Goodlett, R. Aebersold, and R.N. Eisenman. 2002. Quantitative proteomic analysis of Myc oncoprotein function. EMBO J. 21:50885096.

70.Qiu, Y., E.A. Sousa, R.M. Hewick, and J.H. Wang. 2002. Acid-labile isotope-coded extractants: a class of reagents for quantitative mass spectrometric analysis of complex protein mixtures. Anal. Chem. 74:4969-4979.

71.Hughey, C.A., R.P. Rodgers, and A.G. Marshall. 2002. Resolution of $11,000 \mathrm{com}-$ positionally distinct components in a single electrospray ionization Fourier transform ion cyclotron resonance mass spectrum of crude oil. Anal. Chem. 74:4145-4149.

72.Jensen, P.K., L. Paša-Tolić, K.K. Peden, S. Martinovic, M.S. Lipton, G.A. Anderson, N. Tolić, K.-K. Wong, et al. 2000. Mass spectrometric detection for capillary isoelectric focusing separations of complex protein mixtures. Electrophoresis 21:1372-1380.

73.Paša-Tolić, L., M.S. Lipton, C. Masselon, G.A. Anderson, Y. Shen, N. Tolić, and R.D. Smith. 2002. Gene Expression pofiling using advanced mass spectrometric approaches. J. Mass Spectrom. 37:1185-1198.

Received 27 February 2004; accepted 13 May 2004.

Address correspondence to:

Dr. Ljiljana Paša-Tolić

Biological Sciences Division

Pacific Northwest National Laboratory

P.O.B. 999, MSIN: K8-98

Richland, WA, 99352, USA

e-mail: ljiljana.pasatolic@pnl.gov 